\title{
Increased Risk of Stillbirth among Women whose Partner Has Tuberculosis
}

\author{
Qi Sun $\mathbb{D}^{1,2}$ Hongguang Zhang, ${ }^{1}$ Ya Zhang, ${ }^{1}$ Zuoqi Peng, ${ }^{1}$ Jianbo Lu $\mathbb{D},{ }^{1,2}$ and Xu Ma $\mathbb{D}{ }^{1,2}$ \\ ${ }^{1}$ Human Genetics Resource Center, National Research Institute for Family Planning, Beijing 100081, China \\ ${ }^{2}$ Graduate School of Peking Union Medical College \& Chinese Academy of Medical Sciences, Beijing 100730, China \\ Correspondence should be addressed to Jianbo Lu; jblu@lsec.cc.ac.cn and Xu Ma; nfpcc_ma@163.com
}

Received 7 April 2021; Revised 12 July 2021; Accepted 27 August 2021; Published 15 September 2021

Academic Editor: Vincenzo Dario Mandato

Copyright (C) 2021 Qi Sun et al. This is an open access article distributed under the Creative Commons Attribution License, which permits unrestricted use, distribution, and reproduction in any medium, provided the original work is properly cited.

\begin{abstract}
Background. The relationship between tuberculosis (TB) and adverse pregnancy outcomes remains unclear. The aim of our study was to investigate whether TB is a risk factor for adverse pregnancy outcomes including premature birth, low birth weight, and stillbirth. Method. We conducted a population-based retrospective cohort study in mainland China. A total of 3,668,004 Chinese women, along with their partners, were included in this study, within the National Free Pre-Pregnancy Checkups Project, during 2015-2018. Propensity score matching was used to balance the two groups (cases: women or partners with TB; controls: women and partners without TB). Multivariable logistic regression was used to estimate odds ratios (ORs) and 95\% confidence intervals (CIs). Results. Multivariate logistic regression showed that the OR of stillbirth for cases was 1.89 (95\% CI: 1.09-3.16), in comparison with the control group. In the subgroup analysis, women whose partner had TB had a higher risk of stillbirth (OR: 2.13,95\% CI: 1.10-3.86) than women whose partner did not have TB. There was no significant difference in adverse pregnancy outcomes, including preterm birth, low birth weight, and stillbirth, between women with and without TB. Conclusions. Women whose partner had TB were more likely to have stillbirth than women whose partners did not have TB.
\end{abstract}

\section{Background}

Tuberculosis (TB) continues to be a major public health problem, causing 1.5 million deaths in 2018 , and it is the one of the 10 leading causes of death [1]. In 2018, the World Health Organization (WHO) reported that $44 \%$ of TB cases occurred in regions of Southeast Asia, with $9 \%$ of cases being diagnosed in China [2]. TB not only has high mortality, but it also leads to considerable short- and long-term health consequences [3]. Although new drugs and vaccines have been developed to reduce the burden of $\mathrm{TB}$, this disease remains a serious global health concern.

TB mainly affects the lung tissue of patients, but it can also spread to other organs of the body [4]. The third most common form of extrapulmonary TB is female genital TB, which can spread from the lungs through the blood [5]. Mycobacterium tuberculosis affects the endometrium, fallopian tubes, ovaries, and so on, leading to infertility [6]. Studies have found that $\mathrm{TB}$ can lead to adverse pregnancy outcomes, including premature birth, low birth weight
(LBW), stillbirth, and cesarean delivery [6]. However, there are some inconsistencies among the relevant study findings. Recent evidence from the United States suggests that women with TB are more likely to experience postpartum anemia, preterm birth (PTB), and pneumonia than women without TB; in that study, the incidence of maternal respiratory complications were also higher in the TB group than in the control group [7]. A study conducted in Taiwan also showed that women with $\mathrm{TB}$ were at increased risk for adverse pregnancy outcomes compared with controls [8]. Another study found that the percentages of LBW and having a short gestational age were higher among women diagnosed with TB than in women without $\mathrm{TB}$, but there was no significant difference in PTB between the two groups [8]. However, a prospective study in India suggested that there were no statistically significant differences in pregnancy outcomes between women with $\mathrm{TB}$ and controls [9]. These inconsistent results might be owing to the limited sample sizes, insufficient control of confounding factors, and ethnicity in these studies. 
Generally, studies have examined the influence of female factors on adverse pregnancy outcomes; however, little research has been focused on the role of partners in adverse pregnancy outcomes. In our previous study, we found associations of smoking among partners with the prevalence of hypertension among women [10]. Moreover, a case study reported that a healthy asymptomatic woman had been infected with genital TB via sexual intercourse with her partner [11]. Therefore, it is necessary to assess the TB status of partners when investigating the relationship between TB and adverse pregnancy outcomes in women.

The main purpose of this study was to investigate the relationship between $\mathrm{TB}$ in women and adverse pregnancy outcomes in mainland China. A second study aim was to investigate the influence of TB in partners on women's adverse pregnancy outcomes.

\section{Methods}

2.1. Study Population. All data included in this study were obtained from the National Free Pre-Pregnancy Checkups Project (NFPCP). The NFPCP was supported by the National Health and Family Planning Commission and the Ministry of Finance of the People's Republic of China. Free prepregnancy medical examinations and counseling services are provided by the NFPCP to reproductive couples, and most Chinese cities are included in this project. In our study, we included 164 cities in 14 provinces of China from 2015 to 2018 (Figure 1).

All participants included in the NFPCP were asked to complete a structured questionnaire that included information on maternal characteristics, education, alcohol consumption, and smoking. Physical examinations and measurements including weight, height, and blood pressure were conducted by a local doctor. Pregnancy outcomes were obtained during follow-up. TB status was reported by participants in face-to-face interviews.

2.2. Pregnancy Outcomes. Adverse pregnancy outcomes in our study included preterm birth (PTB), low birth weight (LBW), and stillbirth. PTB is defined as an infant born at less than 37 weeks' gestation [12]. LBW is defined as an infant with birth weight less than $2500 \mathrm{~g}$, according to the WHO [13]. Stillbirth is defined as fetal death at or after 20 or 28 weeks of pregnancy [14].

2.3. Covariates. We obtained information on disease history, eating habits, lifestyle, and results of physical and laboratory examinations for pregnant women and their partners. Education was divided into three levels: junior high school and below, high school and junior college, and university and above. Intensity of work was divided into three levels including light, moderate, and heavy, according to participants' occupation. Smoking was defined as smoking two cigarettes a day.

2.4. Statistical Analysis. The Kolmogorov-Smirnov test was used to assess the normality of variables. For continuous variables, $t$-tests or Mann-Whitney $U$ tests were performed, depending on their distribution. The chi-square test was

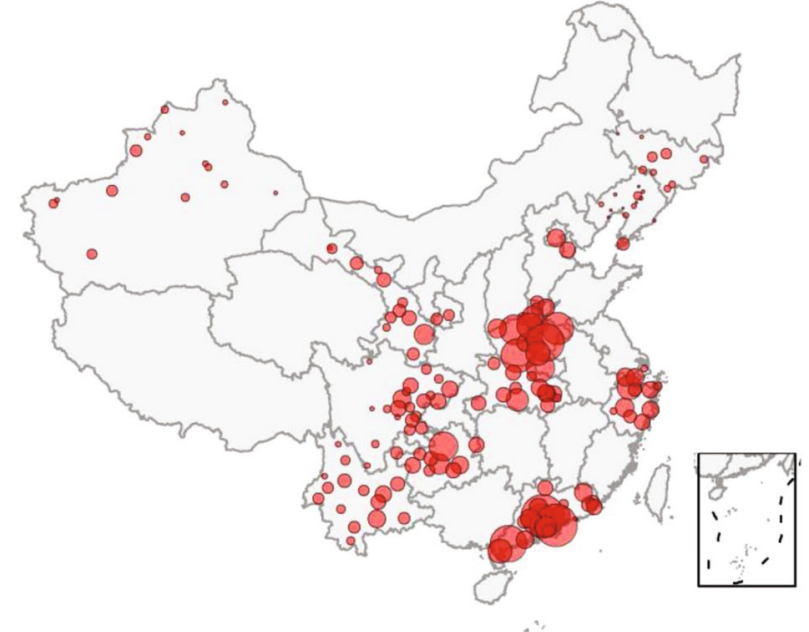

FIgUre 1: The spatial distribution of cities was included in the study (this statistical map was drawn by ourselves using $\mathrm{R}$ software 4.0.0).

used to compare the differences in categorical variables. Propensity score matching (PSM) using body mass index and age was used to balance the two groups. Multiple imputations were applied to impute variables with missing values, with less than $10 \%$ of variables missing. To study the association between TB exposure and adverse pregnancy outcomes, we used a logistic regression model. If the $P$ value of a variable in the univariate analysis was less than 0.05 , the variable was included in the multivariate analysis. All analyses were performed using $\mathrm{R}$ software version 4.0.0 (The R Project for Statistical Computing). A two-sided $P$ value $<0.05$ was considered statistically significant for all statistical tests in our study.

\section{Results}

A total of 3,668,004 women and their partners were included in this study, after excluding individuals with multiple pregnancies, hypertension, heart diseases, diabetes, epilepsy, and chronic nephritis. PSM at a ratio $1: 5$ was used to minimize potential bias owing to unequal distribution between the two groups (cases: women or partners with TB; controls: couples without TB). Details of the study flow are given in Figure 2.

There were significant differences between the case group and the control group with respect to the characteristics of women and partners, including ethnicity, education level, work intensity, residence, and regularity of menstrual cycles (all $P<0.05$ ). There were no significant differences between cases and controls in terms of age at menarche, eating habits, and blood pressure, among others (Table 1). We performed two subgroup analyses (subgroup 1: women with and without TB; subgroup 2: partners with and without TB). The results of the subgroup analyses are shown in Supplementary Table S1.

The outcomes of pregnancy between the case and control groups are shown in Table 2. Higher percentages of PTB $(2.4 \%$ vs. $1.9 \%, P=0.01)$, LBW $(2.0 \%$ vs. $1.5 \%, P<0.01)$, and 


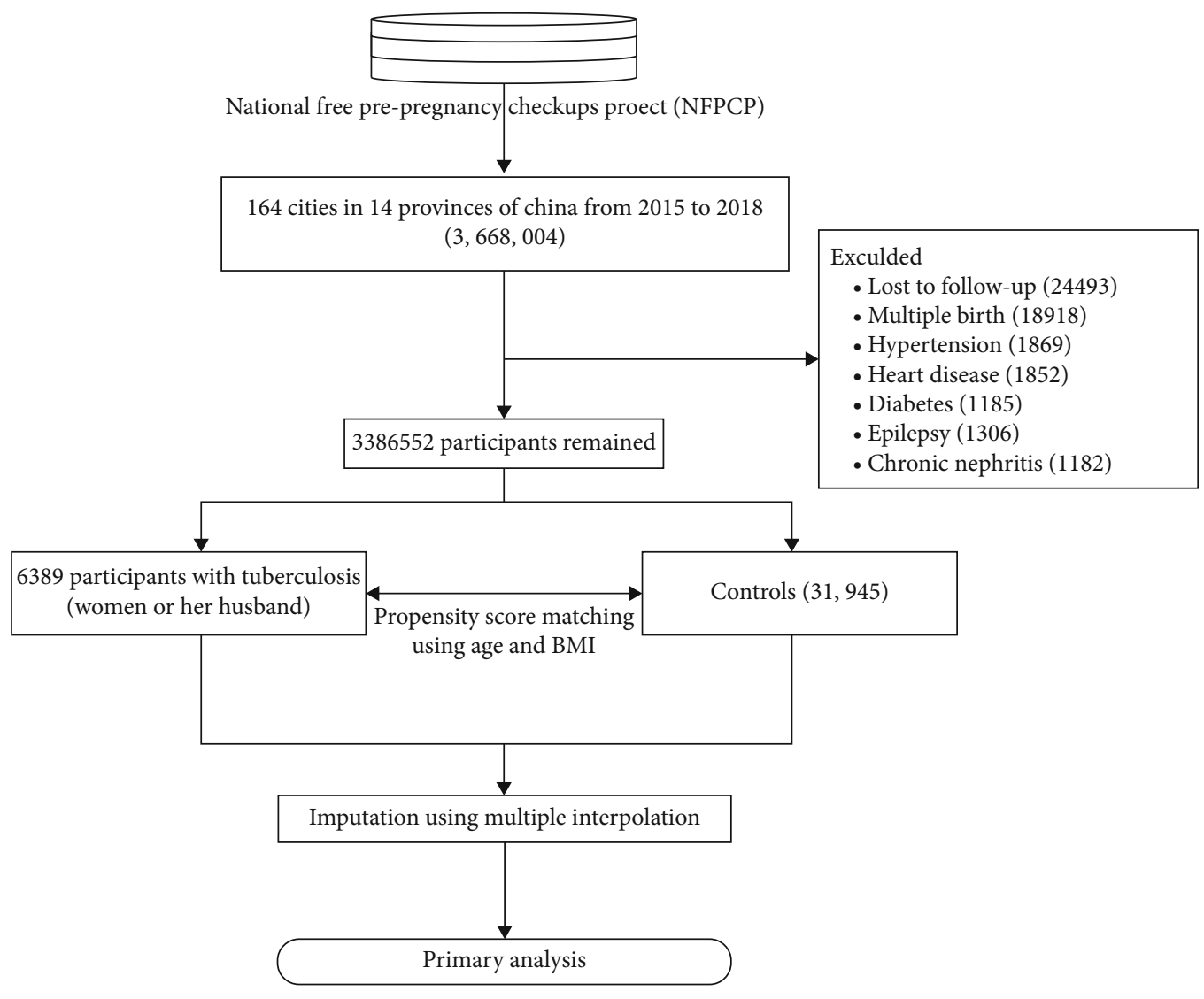

FIGURE 2: Flowchart of our study.

stillbirth $(0.3 \%$ vs. $0.1 \%, P<0.01)$ were observed among cases in comparison with controls (Table 2). Moreover, we identified a significant difference in LBW between women with and without TB and in PTB and stillbirth between partners with and without TB (Supplementary Table S1).

Multivariate logistic regression showed that the odds ratio (OR) of stillbirth for cases was 1.89 (95\% confidence interval (CI): 1.09-3.16), compared with the control group. However, there were no significant differences in PTB and LBW between cases and controls (Table 3 ). In the subgroup analysis, we found no significant differences in PTB (OR: 0.87, 95\% CI: 0.66-1.12), stillbirth (OR: 1.48, 95\% CI: 0.61-3.09), and LBW (OR: 1.04, 95\% CI: 0.79-1.35) between women with and without TB (Table 3). Women whose partner had TB had a higher risk of stillbirth (OR: 2.13, 95\% CI: 1.10-3.86) than women whose partner did not have TB (Table 3). Regarding the treatment of tuberculosis, 39 pregnant women self-reported that they were taking one of the antituberculosis drugs including isoniazid, rifampicin, ethambutol, and pyrazinamide. In order to avoid the influence of drug treatment on pregnancy outcome, we excluded pregnant women undergoing antituberculosis treatment, and the results are consistent with the main analysis (Supplementary Table S2).

\section{Discussion}

This nationwide study included 14 provinces and 164 cities of China, and the sampling sites were located throughout most parts of China. We demonstrated that if a woman or her partner had TB, the woman had a greater risk of stillbirth. Moreover, in subgroup analysis, we found that women whose partner had TB were 2.13 times more likely to have stillbirth than those without TB. However, no effects on PTB, stillbirth, and LBW were found for women with TB.

A cross-sectional retrospective study conducted in Taiwan found no significant differences in PTB between women with and without TB [8]. Tripathy and Tripathy did not observe any adverse pregnancy outcomes in women with TB [9]. Our findings were consistent with those studies in that we found no differences between women with and without TB with respect to adverse pregnancy outcomes, including PTB, LBW, and stillbirth. Our study was based on national data covering the vast majority of the population in China, which makes our research findings convincing.

However, some results in our study were inconsistent with those of previous studies. Some research findings have shown that women with TB have a higher risk of LBW [8], PTB, and perinatal death $[15,16]$. A study conducted in India found that pregnant women with TB had a five times higher risk of PTB than healthy women [17]. There are several possible reasons for the inconsistencies between our research and previous studies including small sample size, few covariates, and different ethnic groups. In this study, our model incorporated lifestyle and dietary habits, disease history, and laboratory test results of pregnant women. Another important reason for the inconsistent results is that 
TABLE 1: Characteristics of the study population between cases and controls.

\begin{tabular}{|c|c|c|c|c|}
\hline Variables & & Control (31945) & Case (6389) & $P$ \\
\hline \multicolumn{5}{|l|}{ Female's characteristics } \\
\hline Age (year) & & $28.00[25.00,31.00]$ & $28.00[25.00,31.00]$ & 0.937 \\
\hline \multirow{2}{*}{ Ethnicity } & Han Chinese & $29398(92.0)$ & $5752(90.0)$ & $<0.001$ \\
\hline & Others & $2547(8.0)$ & $637(10.0)$ & \\
\hline \multirow{3}{*}{ Education level } & Junior high school and below & $15471(48.4)$ & $2064(32.3)$ & $<0.001$ \\
\hline & High school and junior college & $14926(46.7)$ & $3711(58.1)$ & \\
\hline & Bachelor and above & $1548(4.8)$ & $614(9.6)$ & \\
\hline \multirow{3}{*}{ Intensity of work } & Light & $6515(20.4)$ & $2180(34.1)$ & $<0.001$ \\
\hline & Moderate & $4492(14.1)$ & $1401(21.9)$ & \\
\hline & Heavy & $20938(65.5)$ & $2808(44.0)$ & \\
\hline \multirow{2}{*}{ Residence } & Country & $26348(82.5)$ & $4339(67.9)$ & $<0.001$ \\
\hline & City & $5597(17.5)$ & $2050(32.1)$ & \\
\hline Menarche age & & $14.00[13.00,14.00]$ & $13.00[13.00,14.00]$ & 0.54 \\
\hline \multirow{2}{*}{ Has regular menstrual cycle } & No & $1729(5.4)$ & $655(10.3)$ & $<0.001$ \\
\hline & Yes & $30216(94.6)$ & $5734(89.7)$ & \\
\hline \multirow{3}{*}{ Menstrual blood volume } & Much & $819(2.6)$ & $209(3.3)$ & $<0.001$ \\
\hline & Median & $29815(93.3)$ & $5537(86.7)$ & \\
\hline & Few & $1311(4.1)$ & $643(10.1)$ & \\
\hline \multirow{2}{*}{ Dysmenorrhea } & No & $22068(69.1)$ & $3528(55.2)$ & $<0.001$ \\
\hline & Yes & 9877 (30.9) & $2861(44.8)$ & \\
\hline \multirow{3}{*}{ Gravidity } & 0 & $14970(46.9)$ & $2723(42.6)$ & $<0.001$ \\
\hline & 1 & $11946(37.4)$ & $2007(31.4)$ & \\
\hline & $\geq 2$ & $5029(15.7)$ & $1659(26.0)$ & \\
\hline \multirow{2}{*}{ Parity } & 0 & $16916(53.0)$ & $3787(59.3)$ & $<0.001$ \\
\hline & $\geq 1$ & $15029(47.0)$ & $2602(40.7)$ & \\
\hline \multirow{2}{*}{ Eat meat and eggs regularly } & No & $276(0.9)$ & $62(1.0)$ & 0.449 \\
\hline & Yes & $31669(99.1)$ & $6327(99.0)$ & \\
\hline \multirow{2}{*}{ Anorexia vegetables } & No & $31759(99.4)$ & $6349(99.4)$ & 0.743 \\
\hline & Yes & $186(0.6)$ & $40(0.6)$ & \\
\hline \multirow{2}{*}{ Eat raw meat regularly } & No & $31806(99.6)$ & $6330(99.1)$ & $<0.001$ \\
\hline & Yes & $139(0.4)$ & $59(0.9)$ & \\
\hline \multirow{2}{*}{ Smoking } & No & 31854 (99.7) & 6335 (99.2) & $<0.001$ \\
\hline & Yes & $91(0.3)$ & $54(0.8)$ & \\
\hline \multirow{2}{*}{ Passive smoking } & No & $27747(86.9)$ & $4596(71.9)$ & $<0.001$ \\
\hline & Yes & $4198(13.1)$ & $1793(28.1)$ & \\
\hline \multirow{2}{*}{ Drinking } & No & $30697(96.1)$ & $5772(90.3)$ & $<0.001$ \\
\hline & Yes & $1248(3.9)$ & $617(9.7)$ & \\
\hline \multirow{4}{*}{ Pressure of life and work } & Never & $24511(76.7)$ & 3827 (59.9) & $<0.001$ \\
\hline & Mild & $3994(12.5)$ & $922(14.4)$ & \\
\hline & Moderate & $3166(9.9)$ & $1430(22.4)$ & \\
\hline & Severe & $274(0.9)$ & $210(3.3)$ & \\
\hline BMI $\left(\mathrm{kg} / \mathrm{m}^{2}\right)$ & & $20.57[19.04,22.67]$ & $20.56[19.03,22.67]$ & 0.77 \\
\hline SBP (mmHg) & & $110.00[100.00,115.00]$ & $110.00[100.00,116.00]$ & 0.511 \\
\hline DBP (mmHg) & & $70.00[66.00,75.00]$ & $70.00[65.00,76.00]$ & 0.441 \\
\hline \multirow{4}{*}{ Blood group } & $\mathrm{O}$ & $10736(33.6)$ & $2370(37.1)$ & $<0.001$ \\
\hline & A & $9279(29.0)$ & $1807(28.3)$ & \\
\hline & $\mathrm{B}$ & $9122(28.6)$ & $1738(27.2)$ & \\
\hline & $\mathrm{AB}$ & $2808(8.8)$ & $474(7.4)$ & \\
\hline
\end{tabular}


TABle 1: Continued.

\begin{tabular}{|c|c|c|c|c|}
\hline Variables & & Control (31945) & Case (6389) & $P$ \\
\hline \multirow{2}{*}{$\mathrm{Rh}$ blood group } & Po & $31843(99.7)$ & $6373(99.7)$ & 0.433 \\
\hline & $\mathrm{Ne}$ & $102(0.3)$ & $16(0.3)$ & \\
\hline $\mathrm{FBG}(\mathrm{mmol} / \mathrm{L})$ & & $4.78[4.32,5.20]$ & $4.81[4.40,5.21]$ & $<0.001$ \\
\hline $\operatorname{ALT}(\mathrm{U} / \mathrm{L})$ & & $16.00[11.70,22.90]$ & $15.00[11.00,21.00]$ & $<0.001$ \\
\hline Creatinine $(\mu \mathrm{mol} / \mathrm{L})$ & & $63.00[53.00,74.00]$ & $59.00[51.00,70.00]$ & $<0.001$ \\
\hline $\mathrm{TSH}(\mathrm{mIU} / \mathrm{L})$ & & $1.61[1.07,2.34]$ & $1.78[1.15,2.65]$ & $<0.001$ \\
\hline \multicolumn{5}{|l|}{ History of diseases in female } \\
\hline \multirow{2}{*}{ Anemia } & No & $31355(98.2)$ & $6133(96.0)$ & $<0.001$ \\
\hline & Yes & $590(1.8)$ & $256(4.0)$ & \\
\hline \multirow{2}{*}{ Thyroid disease } & No & $31739(99.4)$ & $6280(98.3)$ & $<0.001$ \\
\hline & Yes & $206(0.6)$ & $109(1.7)$ & \\
\hline \multirow{2}{*}{ History of premature birth } & No & $31867(99.8)$ & $6364(99.6)$ & 0.052 \\
\hline & Yes & $78(0.2)$ & $25(0.4)$ & \\
\hline \multirow{2}{*}{ History of stillbirth } & No & $31693(99.2)$ & $6294(98.5)$ & $<0.001$ \\
\hline & Yes & $252(0.8)$ & $95(1.5)$ & \\
\hline \multirow{2}{*}{ History of natural abortion } & No & $30929(96.8)$ & $5956(93.2)$ & $<0.001$ \\
\hline & Yes & $1016(3.2)$ & $433(6.8)$ & \\
\hline \multirow{3}{*}{ History of artificial abortion } & 0 & $26664(83.5)$ & $4354(68.1)$ & $<0.001$ \\
\hline & 1 & $3605(11.3)$ & $1324(20.7)$ & \\
\hline & $>1$ & $1676(5.2)$ & $711(11.1)$ & \\
\hline \multicolumn{5}{|l|}{ Partner's characteristics } \\
\hline Age (year) & & $29.00[26.00,33.00]$ & $30.00[27.00,34.00]$ & $<0.001$ \\
\hline \multirow{2}{*}{ Ethnicity } & Han & $29537(92.5)$ & $5812(91.0)$ & $<0.001$ \\
\hline & Other & $2408(7.5)$ & $577(9.0)$ & \\
\hline \multirow{3}{*}{ Education level } & Junior high school and below & $15187(47.5)$ & $1992(31.2)$ & $<0.001$ \\
\hline & High school and junior college & $15185(47.5)$ & $3777(59.1)$ & \\
\hline & Bachelor and above & $1573(4.9)$ & $620(9.7)$ & \\
\hline \multirow{3}{*}{ Intensity of work } & Light & $4974(15.6)$ & $1747(27.3)$ & $<0.001$ \\
\hline & Moderate & $5454(17.1)$ & $1651(25.8)$ & \\
\hline & Heavy & $21517(67.4)$ & $2991(46.8)$ & \\
\hline \multirow{2}{*}{ Eat meat and eggs regularly } & No & $323(1.0)$ & $86(1.3)$ & 0.021 \\
\hline & Yes & $31622(99.0)$ & $6303(98.7)$ & \\
\hline \multirow{2}{*}{ Anorexia vegetables } & No & $31765(99.4)$ & $6304(98.7)$ & $<0.001$ \\
\hline & Yes & $180(0.6)$ & $85(1.3)$ & \\
\hline \multirow{2}{*}{ Eat raw meat } & No & $31734(99.3)$ & $6299(98.6)$ & $<0.001$ \\
\hline & Yes & $211(0.7)$ & $90(1.4)$ & \\
\hline \multirow{2}{*}{ Smoking } & No & $22878(71.6)$ & $4182(65.5)$ & $<0.001$ \\
\hline & Yes & $9067(28.4)$ & $2207(34.5)$ & \\
\hline \multirow{2}{*}{ Passive smoking } & No & $23041(72.1)$ & $3487(54.6)$ & $<0.001$ \\
\hline & Yes & $8904(27.9)$ & $2902(45.4)$ & \\
\hline \multirow{2}{*}{ Drinking } & No & 22007 (68.9) & $3266(51.1)$ & $<0.001$ \\
\hline & Yes & $9938(31.1)$ & $3123(48.9)$ & \\
\hline \multirow{4}{*}{ Pressure of life and work } & Never & $23371(73.2)$ & $3489(54.6)$ & $<0.001$ \\
\hline & Mild & $4723(14.8)$ & $960(15.0)$ & \\
\hline & Moderate & $3370(10.5)$ & $1627(25.5)$ & \\
\hline & Severe & $481(1.5)$ & $313(4.9)$ & \\
\hline BMI $\left(\mathrm{kg} / \mathrm{m}^{2}\right)$ & & $22.86[21.00,25.05]$ & $22.49[20.42,24.77]$ & $<0.001$ \\
\hline $\mathrm{SBP}(\mathrm{mmHg})$ & & $118.00[110.00,121.00]$ & $118.00[110.00,124.00]$ & $<0.001$ \\
\hline
\end{tabular}


TABLE 1: Continued.

\begin{tabular}{|c|c|c|c|c|}
\hline Variables & & Control (31945) & Case (6389) & $P$ \\
\hline DBP (mmHg) & & $75.00[70.00,80.00]$ & $75.00[70.00,80.00]$ & 0.091 \\
\hline \multirow{4}{*}{ Blood group } & $\mathrm{O}$ & $10861(34.0)$ & $2335(36.5)$ & $<0.001$ \\
\hline & A & $9280(29.0)$ & $1870(29.3)$ & \\
\hline & B & $9003(28.2)$ & $1705(26.7)$ & \\
\hline & $\mathrm{AB}$ & $2801(8.8)$ & $479(7.5)$ & \\
\hline \multirow{2}{*}{ Rh blood group } & Po & 31839 (99.7) & $6375(99.8)$ & 0.177 \\
\hline & $\mathrm{Ne}$ & $106(0.3)$ & $14(0.2)$ & \\
\hline ALT (U/L) & & $25.00[17.60,36.00]$ & $25.00[17.00,38.20]$ & 0.114 \\
\hline Creatinine $(\mu \mathrm{mol} / \mathrm{L})$ & & $81.00[72.40,91.00]$ & $81.20[72.50,91.50]$ & 0.484 \\
\hline
\end{tabular}

The distribution of continuous variables is expressed as the median [lower quartile, upper quartile]. BMI: body mass index; SBP: systolic blood pressure; DBP: diastolic blood pressure; FBG: fasting blood glucose; ALT: alanine aminotransferase; TSH: thyroid stimulating hormone; Ne: negative; Po: positive; case: women or partner with TB; control: healthy women with healthy partner.

TABLE 2: The outcomes of pregnancy in cases and controls.

\begin{tabular}{lcccc}
\hline Variables & & Control $(31945)$ & Case $(6389)$ & $P$ \\
\hline Gestational weeks & & $39.00[38.00,40.00]$ & $39.00[38.00,40.00]$ & $6233(97.6)$ \\
Premature birth & No & $31326(98.1)$ & $156(2.4)$ & 0.001 \\
& Yes & $619(1.9)$ & $6261(98.0)$ & 0.009 \\
LBW & No & $31453(98.5)$ & $6369(99.7)$ & $20(0.3)$ \\
Yes & No & $492(1.5)$ & $3317(51.9)$ & 0.006 \\
Stillbirth & Yes & $31898(99.9)$ & $3072(48.1)$ & 0.525 \\
Birth gender & Man & $47(0.1)$ & $3250.00[3000.00,3500.00]$
\end{tabular}

LBW: low birth weight; the distribution of continuous variables is expressed as the median [lower quartile, upper quartile]; case: women or partner with TB; control: healthy women with healthy partner.

TABLE 3: Adjusted odds ratio estimates and 95\% confidence intervals of PTB, stillbirth, and LBW for each group.

\begin{tabular}{lccr}
\hline & PTB (adjusted OR 95\% CI) & Stillbirth (adjusted OR 95\% CI) $^{\mathrm{b}}$ & LBW (adjusted OR 95\% CI) $^{\mathrm{c}}$ \\
\hline Women or partner with TB & $0.97(0.80-1.16)$ & $1.89(1.09-3.16)$ & $0.97(0.79-1.19)$ \\
TB women with healthy partner & $0.87(0.66-1.12)$ & $1.48(0.61-3.09)$ & $1.04(0.79-1.35)$ \\
Healthy women with TB partner & $1.03(0.81-1.29)$ & $2.13(1.10-3.86)$ & $0.89(0.67-1.16)$ \\
\hline
\end{tabular}

PTB: preterm birth; LBW: low birth weight; OR: odds ratio; CI: confidence interval. ${ }^{a}$ Model adjusted for female's characteristics (age, ethnicity, education level, intensity of work, residence, has regular menstrual cycle, dysmenorrhea, gravidity, eat meat and eggs regularly, passive smoking, drinking, pressure of life and work, BMI, and creatinine), history of diseases in female (anemia, history of premature birth, history of natural abortion, and history of artificial abortion), and husband's characteristics (age, ethnicity, education level, intensity of work, eat raw meat, smoking, passive smoking, drinking, pressure of life and work, SBP (mmHg), blood group, and ALT). 'Model adjusted for female's characteristics (ethnicity and dysmenorrhea), history of stillbirth, and husband's characteristics (age, ethnicity, SBP, and DBP). 'Model adjusted for female's characteristics (age, ethnicity, education level, intensity of work, residence, has regular menstrual cycle, menstrual blood volume, dysmenorrhea, passive smoking, drinking, pressure of life and work, BMI, and creatinine), history of diseases in female (anemia, history of premature birth, and history of natural abortion), and husband's characteristics(age, education level, intensity of work, eat raw meat, smoking, passive smoking, drinking, pressure of life and work, SBP, blood group, and ALT).

previous studies have not taken into account the effect of a woman's partner on pregnancy outcomes. Our previous study showed that this is important as we found an effect of the partner's smoking on spontaneous abortion [18].

The other main finding of the present study is that having a partner with TB was found to be a risk factor for stillbirth in pregnant women, which has not been reported previously. One case report demonstrated that $\mathrm{TB}$ can be transmitted from partner to his wife via semen [19]. Another study showed that female genital TB could influence endometrial metabolism [20]. These metabolic changes might be associated with stillbirth in pregnant women. Animal experiments have demonstrated that TB affects the histophysiology of the male reproductive system [21]. Another explanation for our result is that $\mathrm{TB}$ affects the quality of sperm, which could lead to stillbirth. 
Previous studies have shown that TB treatment can reduce the risk of TB to pregnant women and fetuses [22]. Further studies found that TB treatment in the first trimester can reduce the risk of preterm birth, low birth weight, and fetal death [23]. In our study, antituberculosis drug treatment did not affect the pregnancy outcome of pregnant women. The possible reason for this result is that too few pregnant women in the cohort self-reported taking antituberculosis drugs. A possible reason for the low number of people taking antituberculosis drugs in our study was that all TB patients are assessed for suitability for pregnancy before conception. If a woman was taking antituberculosis drugs, her doctor may recommend delaying pregnancy until antituberculosis treatment was completed.

There were several limitations in this study that should be noted. First, in our study, TB status was self-reported, which made it impossible to confirm whether the participant's TB was active or not. Second, owing to self-reporting, some patients with TB might have concealed their medical history, which may lead to selection bias. Third, in the discussion, we hypothesized that the partner could transmit TB via semen, but we did not collect the quality of the partner's sperm, clinical condition of the partner at conception, and how long the couples have been in contact, which limited the reliability of our conjecture. Fourth, anti-TB treatment has an impact on pregnancy outcomes, but too few patients in our study were taking anti-TB drugs, which may have affected the results.

\section{Conclusion}

Among the pregnant women in our study, having TB was not a risk factor for LBW, PTB, and stillbirth. Women whose partners had $\mathrm{TB}$ were more likely to have stillbirth than women whose partners did not have TB.

\section{Data Availability}

The datasets used and analyzed during the current study are available from the corresponding authors on reasonable request.

\section{Ethical Approval}

This retrospective cohort study was approved by the Institutional Research Review Board at National Research Institute for Family Planning and performed in accordance with the ethical standards laid down in the 1964 Declaration of Helsinki. The data used in our study acquired administrative permissions.

\section{Disclosure}

The funder did not have any role in the design, collection, analysis, or interpretation of data or in the drafting of the manuscript.

\section{Conflicts of Interest}

The authors declare that there is no conflict of interest regarding the publication of this article.

\section{Authors' Contributions}

$\mathrm{XM}$ and JBL conceived and designed the work. HGZ managed and sorted out the data. YZ performed the analysis. QS was a major contributor in writing the manuscript. ZQP and XM reviewed and edited the manuscript. All authors read and approved the final manuscript.

\section{Acknowledgments}

This work was supported by the National Key Research and Development Program of China (2016YFC1000307) and the subproject of the National Key Research and Development Program of China (2016YFC1000307-10).

\section{Supplementary Materials}

Supplementary 1. Table S1 comparison of characteristics and pregnancy outcomes among couples with different conditions of TB.

Supplementary 2. Table S2 adjusted odds ratio estimates and 95\% confidence intervals of PTB, stillbirth, and LBW in pregnant women without TB treatment.

\section{References}

[1] Organization WH, Tuberculosis2020 https://www.who.int/ news-room/fact-sheets/detail/tuberculosis.

[2] Organization WH, Global tuberculosis report 20192019 https:// www.who.int/tb/publications/global_report/en/.

[3] M. Shah and C. Reed, "Complications of tuberculosis," Current Opinion in Infectious Diseases, vol. 27, no. 5, pp. 403410, 2014.

[4] O. M. Loto and I. Awowole, "Tuberculosis in pregnancy: a review," Journal of Pregnancy, vol. 2012, Article ID 379271, 2012.

[5] S. Gupta and P. Gupta, "Etiopathogenesis, challenges and remedies associated with female genital tuberculosis: potential role of nuclear receptors," Frontiers in Immunology, vol. 11, article 02161, 2020.

[6] A. Muneer, B. Macrae, S. Krishnamoorthy, and A. Zumla, "Urogenital tuberculosis - epidemiology, pathogenesis and clinical features," Nature Reviews. Urology, vol. 16, no. 10, pp. 573-598, 2019.

[7] A. El-Messidi, N. Czuzoj-Shulman, A. R. Spence, and H. A. Abenhaim, "Medical and obstetric outcomes among pregnant women with tuberculosis: a population-based study of 7.8 million births," American journal of obstetrics and gynecology, vol. 215, no. 6, pp. 797.e1-797.e6, 2016.

[8] H. C. Lin, H. C. Lin, and S. F. Chen, "Increased risk of low birthweight and small for gestational age infants among women with tuberculosis," BJOG : An International Journal of Obstetrics and Gynaecology, vol. 117, no. 5, pp. 585-590, 2010. 
[9] S. N. Tripathy and S. N. Tripathy, "Tuberculosis and pregnancy," International Journal of Gynaecology and Obstetrics, vol. 80, no. 3, pp. 247-253, 2003.

[10] Y. Yang, F. Liu, L. Wang et al., “Association of husband smoking with wife's hypertension status in over 5 million Chinese females aged 20 to 49 years," Journal of the American Heart Association, vol. 6, no. 3, 2017.

[11] M. Kimura, H. Araoka, H. Baba et al., "First case of sexually transmitted asymptomatic female genital tuberculosis from spousal epididymal tuberculosis diagnosed by active screening," International Journal of Infectious Diseases, vol. 73, pp. 60-62, 2018.

[12] A. Dbstet, "WHO: recommended definitions, terminology and format for statistical tables related to the perinatal period and use of a new certificate for cause of perinatal deaths. Modifications recommended by FIGO as amended October 14, 1976," Acta Obstetricia et Gynecologica Scandinavica, vol. 56, no. 3, pp. 247-253, 1977.

[13] Organization WH, Global nutrition targets 2025: low birth weight policy brief, World Health Organization, 2014.

[14] R. M. Silver, M. W. Varner, U. Reddy et al., "Work-up of stillbirth: a review of the evidence," American journal of obstetrics and gynecology., vol. 196, no. 5, pp. 433-444, 2007.

[15] R. Figueroa-Damian and J. L. Arredondo-Garcia, "Neonatal outcome of children born to women with tuberculosis," Archives of Medical Research, vol. 32, no. 1, pp. 66-69, 2001.

[16] N. Jana, K. Vasishta, S. K. Jindal, B. Khunnu, and K. Ghosh, "Perinatal outcome in pregnancies complicated by pulmonary tuberculosis," International Journal of Gynaecology and Obstetrics, vol. 44, no. 2, pp. 119-124, 1994.

[17] S. Chopra, S. Siwatch, N. Aggarwal, P. Sikka, and V. Suri, "Pregnancy outcomes in women with tuberculosis: a 10-year experience from an Indian tertiary care hospital," Tropical Doctor, vol. 47, no. 2, pp. 104-109, 2017.

[18] L. Wang, Y. Yang, F. Liu et al., "Paternal smoking and spontaneous abortion: a population-based retrospective cohort study among non-smoking women aged 20-49 years in rural China," Journal of Epidemiology and Community Health, vol. 72, no. 9, pp. 783-789, 2018.

[19] J. K. Lattimer, H. P. Colmore, G. Sanger, D. H. Robertson, and L. F. Mc, "Transmission of genital tuberculosis from husband to wife via the semen," American Review of Tuberculosis, vol. 69, no. 4, pp. 618-624, 1954.

[20] E. Subramani, M. Jothiramajayam, M. Dutta et al., "NMRbased metabonomics for understanding the influence of dormant female genital tuberculosis on metabolism of the human endometrium," Human Reproduction, vol. 31, no. 4, pp. 854865, 2016.

[21] B. Ramos Robles, R. A. Valdez, U. J. Hernández et al., "Immunoendocrine abnormalities in the male reproductive system during experimental pulmonary tuberculosis," Tuberculosis (Edinburgh, Scotland), vol. 109, pp. 109-116, 2018.

[22] K. Miele, S. Bamrah Morris, and N. K. Tepper, "Tuberculosis in pregnancy," Obstetrics and Gynecology, vol. 135, no. 6, pp. 1444-1453, 2020.

[23] S. Sobhy, Z. Babiker, J. Zamora, K. S. Khan, and H. Kunst, "Maternal and perinatal mortality and morbidity associated with tuberculosis during pregnancy and the postpartum period: a systematic review and meta-analysis," BJOG : An International Journal of Obstetrics and Gynaecology, vol. 124, no. 5, pp. 727-733, 2017. 